\title{
ESTILOS DE PENSAR E CRIAR NO CONTEXTO ORGANIZACIONAL: DIFERENÇAS DE ACORDO COM O CARGO PROFISSIONAL?
}

\author{
Tatiana de Cássia Nakano \\ Doutora em Psicologia, docente do programa de pós-graduação stricto sensu em Psicologia da \\ PUC-Campinas. \\ Carolina Rosa Campos \\ Mestranda do curso de pós-graduação em Psicologia da PUC-Campinas, bolsista CNPq.
}

Talita Fernanda da Silva Mestranda do curso de pós-graduação em Psicologia da PUC-Campinas, bolsista CNPq.

Elida Kalina Gomes Pereira

Psicóloga graduada pelo Centro Universitário de João Pessoa.

\begin{abstract}
Resumo
O presente estudo visou identificar o estilo de pensar e criar dos profissionais de uma empresa do ramo de distribuição de produtos farmacêuticos, a fim de verificar a existência de diferentes estilos predominantes, de acordo com as variáveis sexo, escolaridade e cargo ocupado. Quarenta profissionais, sendo 30 mulheres e 10 homens, com idades entre 17 anos e 35 anos $(M=24,5 ; D P=5,4)$ e escolaridade correspondente ao Ensino Médio $(n=14)$ e Superior $(n=26)$ foram divididos em dois grupos, sendo o primeiro formado por profissionais que trabalhavam na área fiscal e contábil $(n=20)$, e outro grupo de profissionais que exerciam cargos na área de vendas $(n=20)$. Os participantes responderam à Escala de Estilos de Pensar e Criar de forma individual durante o horário de trabalho. Os resultados demonstraram que somente o estilo lógico-objetivo mostrou-se influenciado pela área de atuação $(F=4,745 ; \quad p \leq 0,037)$, sendo que todas as demais influências não mostraram significativas. No presente estudo, as variáveis sexo e nível de escolaridade não exerceram influência nos estilos de pensar e criar dos participantes, assim como todas as demais interações. Verificou-se ainda que a maior parte da amostra estudada apresenta como estilo predominante o Lógico-objetivo, independente do sexo (62,0\% das mulheres foram classificadas nesse estilo e $72,7 \%$ dos homens), do nível de escolaridade (com 57,1\% dos profissionais com Ensino Médio e 69,2\% com Ensino Superior) e em relação à área de atuação $(60,0 \%$ dos participantes da área contábil/fiscal apresentando esse estilo predominante e 70,0\% dos profissionais da área de vendas).
\end{abstract}

Palavras-chave: estilos, criatividade, gênero, escolaridade, organizacional. 


\title{
STYLES OF THINKING AND CREATING IN ORGANIZATIONAL CONTEXT: DIFFERENCES ACCORDING TO PROFESSIONAL POSITION?
}

\begin{abstract}
This study aimed to identify the style of thinking and creating in professionals a pharmaceutical distribution company, in order to verify the existence of different styles according to gender, educational level and position held. Forty professionals, including 30 women and 10 men, aged 17 years and 35 years $(M=24.5, S D=5.4)$, with high school education $(n=14)$ and college $(n=26)$ were divided into two groups, the first being formed by professionals working in the tax and accounting $(n=20)$, and another group of professionals who hold positions in the sales $(n=20)$. They answered the scale Styles of Thinking and Creating individually during office hours. The results showed that only the logical-objective style proved to be influenced by the area of performance $(F=4.745 ; p \leq 0.037)$, with all others variables showed no significant influences. In the present study, gender and educational level did not influence the styles of thinking and creating of the participants as well as all other interactions. It was also found that most of the sample appears as the predominant style logical-objective, regardless of sex $(62.0 \%$ of women and $72.7 \%$ of men were classified in this style), level of education (with $571 \%$ of professionals with high school and $69.2 \%$ with college) and in relation to the field $(60.0 \%$ of the participants in the accounting/tax filing this predominant style and $70.0 \%$ of professionals in sales).
\end{abstract}

Keywords: style, creativity, gender, education, organizational.

\section{ESTILOS DE PENSAR Y DE CREAR EN EL CONTEXTO DE LA ORGANIZACIÓN: DIFERENCIAS DE ACUERDO A LA POSICIÓN PROFESIONAL?}

\begin{abstract}
Resumen
Este estudio tuvo como objetivo identificar el estilo de pensamiento y de creación de los profesionales de una empresa de distribución farmacéutica con el fin de verificar la existencia de estilos diferentes en función del sexo, nivel educativo y cargo. Cuarenta profesionales, 30 mujeres y 10 hombres, de 17 años y 35 años $(M=24,5, S D=5,4)$ con la educación que corresponde la escuela secundaria $(n=14)$ y superior $(n=26)$ fueron divididos en dos grupos, siendo el primero formado por los profesionales que trabajan en los impuestos y contabilidad $(n=20)$, y otro grupo de profesionales que ocupan cargos en el área de ventas $(n=20)$. Los participantes respondieron a Escala de Estilos de Pensar y Crear de forma individual durante las horas de trabajo. Los resultados mostraron que sólo el estilo logico-objetivo resultó ser influenciados por el área de desempeño $(F=4,745, p \leq 0,037)$, y todos los demás no mostraron una influencia. En el presente estudio, sexo y nivel educativo mostró que no influyen en los estilos de pensamiento y la creación de los participantes, así como todas las demás interacciones. También se encontró que la mayoría de la muestra aparece como el estilo predominante lógico-objetivo, independientemente de su sexo $(62,0 \%$ de las mujeres y $72,7 \%$ de los hombres fueran classificadas en este estilo), nivel de educación (con $571 \%$ de los profesionales de la escuela secundaria y el $69,2 \%$ con educación superior) y en relación con el campo $(60,0 \%$ de los participantes en la presentación de la contabilidad/impuestos de este estilo predominante y el 70,0\% de los profesionales en las ventas).
\end{abstract}

Palabras claves: estilo, creatividad, sexo, educación, organización. 


\section{INTRODUÇÃO}

A sociedade vivencia um momento histórico marcado por intensas mudanças, cujo cenário tem estimulado e valorizado as expressões criativas (Nicolas, 1999; Oliveira, 2007). Vista como um importante recurso individual para lidar com os desafios da época atual (Gonçalves, Fleith \& Libório, 2011), a criatividade tem sido tema de estudos em diferentes áreas do conhecimento e nos variados campos de sua ocorrência (Bruno-Faria, Veiga \& Macedo, 2008; Zanella \& Titon, 2005).

Dentre os diferentes contextos sociais, destaque deve ser dado à sua valorização nos contextos organizacionais, locais onde o construto volta-se principalmente para os setores de gestão, recursos humanos, marketing, entre outros, dada sua possibilidade de atuar enquanto processo que pode gerar inovação e ganhos, tanto para a empresa, como para o funcionário (Nakano \& Wechsler, 2007; Sakamoto, 2000; Wechsler, 2001). Nesses locais, sua importância ampara-se no fato de que estudos que envolvem a criatividade, nesse contexto, podem auxiliar as organizações a lidar com desafios de forma inovadora (Moraes \& Lima, 2009), capacitando-as a responder às exigências do mercado de trabalho, principalmente aquelas voltadas à necessidade de contratação de profissionais que se mostrem competentes e capazes de agir, de forma inovadora e criativa, frente a diferentes situações (Oliveira, 2010). Assim, o contexto organizacional passa por um processo no qual tem sido valorizado, cada vez mais, o "desenvolvimento e seleção profissional especializada, de pessoas adaptáveis e flexíveis, capazes de ter sucesso em contextos desafiantes, complexos e em constantes mudanças" (Candeias, Rebelo, Silva \& Mendes, 2011, p.54).

Vale ressaltar o papel fundamental que o ambiente exerce na expressão criativa do indivíduo, tanto para a emergência como para a repressão da criatividade (Alencar, 2001; De La Torre, 2005; Wechsler, 2008). Nesse sentido, pode-se dizer que as organizações enfrentam atualmente alguns desafios, tais como a superação da resistência a idéias novas, a necessidade de desenvolvimento de mudanças em relação aos possíveis conflitos provenientes das relações interpessoais e do clima organizacional, de forma que promover a conscientização dos funcionários quanto à sua capacidade de criação, consequentemente estimulando e valorizando a criatividade dos mesmos, tornou-se essencial para a sobrevivência organizacional (Alencar, 2005; Oliveira, 
2010). Como consequência, tal característica passou a ser exigida pelas empresas, cada vez mais, como elemento fundamental e/ou requisito para que um indivíduo possa se candidatar ou preencher uma vaga (Lins \& Miyata, 2008).

No entanto, embora o número de estudos acerca do construto venham ganhado espaço no meio científico nas últimas décadas (El-Murad \& West, 2004; Nakano, 2005; Wechsler \& Nakano, 2002, 2003), ainda são poucas as pesquisas que abordam a sua aplicação no contexto organizacional, voltadas à investigação dos fatores influenciadores do potencial criativo dos funcionários e de instrumentos que possam avaliá-los (Crespo, 2004; Nakano \& Wechsler, 2007; Zanella \& Titon, 2005). Em uma análise da produção científica nacional, BrunoFaria, Veiga e Macêdo (2008) constataram que esta área encontra-se em um estado inaugural, visto que a maior parte dos trabalhos existentes constituem-se em manuais "práticos" de como tornar-se mais criativo, sendo raros os estudos científicos sobre o tema.

$\mathrm{Na}$ literatura, dentre os poucos estudos existentes, é possível verificar pesquisas que têm como foco a avaliação das barreiras à criatividade nos ambientes organizacionais, cujos resultados têm demonstrado aspectos como pressão para criar, clima prejudicado de diálogo e de estímulo à participação, bem como falta de estrutura organizacional flexível (Alencar \& Fleith, 2003). Por outro lado, também se fazem presentes trabalhos que voltados ao conhecimento de características favorecedoras da criatividade, as quais constituem-se, principalmente, em práticas de valorização das pessoas e de promoção de condições para que cada trabalhador procure dar o melhor de si, baseadas na promoção da autonomia, percepção de abertura por parte da empresa e boa recepção a possíveis mudanças que sejam benéficas à organização como um todo (Alencar, 2005). Da mesma forma, Correia e Dellagnelo (2004) salientam estruturas flexíveis e inovadoras como estímulos ao potencial criativo das organizações. Entretanto, uma crítica que se faz presente refere-se ao fato de que, muitas vezes, os programas de incentivo criativo organizacional acabam sendo elaborados de forma generalizada, de forma a desconsiderar as diferenças individuais de personalidade ou das características próprias do grupo. Nesse sentido, no tocante à diversidade dos ambientes de trabalho, pode-se citar, como exemplo, a pressão para criar bastante enfatizada na área de propaganda e marketing, na qual a atividade de criar coisas novas é uma rotina (Bruno-Faria, Veiga \& Macêdo, 2008). Assim, Wechsler (2006b) enfatiza que, 
independentemente da pressão social, as pessoas criativas apresentam algumas características bem peculiares, de forma que a tentativa de identificar tendências de comportamento e sentimento nessas pessoas pode revelar importantes informações sobre a criatividade, destacando-se, dentre as possibilidades, a importância do conhecimento e identificação dos estilos de pensar e criar, foco da presente pesquisa.

No contexto organizacional, conforme salientado por Martinez (2000), três focos devem ser trabalhados considerando-se a complexidade da criatividade, sendo eles os indivíduos, grupos e a organização como um todo, de maneira que, segundo a autora, devem ser definidas e desenvolvidas ações em função da necessidade da organização, sempre visando a promoção da criatividade desses três elementos. Entretanto, o que se nota é que, enquanto ideal a ser seguido, na prática não é essa situação que é comum de se encontrar dentro das empresas. Embora os estudos venham demonstrando que, no âmbito das organizações, a criatividade capacita os funcionários a inovar e solucionar novos problemas (Alencar, 2005; Martinez, 2002), o que se verifica é que, nesse contexto, a criatividade não vem sendo estimulada, dado o fato de que, apesar de alguns líderes (ou gerentes) reconhecerem a importância da criatividade e do valor das idéias novas, o ambiente de trabalho ainda continua priorizando a subordinação, a produtividade tradicional e o controle, conforme salientado por Mundim e Wechsler (2007).

Ainda em relação à sua utilização no contexto organizacional, Wechsler (2001) destaca que, embora muitas pessoas ingressem no mercado de trabalho com alto potencial criativo, as mesmas não encontram apoio ou reconhecimento por suas idéias criativas no seu ambiente profissional. Assim, contraditoriamente ao fato de que, embora se faça presente, atualmente, uma busca por talentos criativos nas empresas, e consequentemente um crescente interesse por pesquisas que visem a identificação do perfil de líderes criativos e formas mais inovadoras para se atuar no mercado de trabalho, os resultados de pesquisas têm apontado, segundo a autora, para a existência de um descompasso entre o desejo de se possuir líderes criativos nas empresas, e as reais condições oferecidas por estas para o cultivo e a implementação de novas idéias, de forma que se faz necessário o desenvolvimento de mais trabalhos em nível empresarial. Nesse sentido, o que se pode verificar é que as empresas possivelmente ainda não perceberam que "a existência de pessoas criativas aumenta as possibilidades 
de sobrevivência da mesma, nos momentos de crise e mudança, já que pode contar com profissionais suficientemente criativos e flexíveis para perceberem o melhor rumo a dar à organização" (Candeias e colaboradores, 2001, p.75).

Também Alencar (2005) afirma que cabe ao líder estar atento às necessidades da equipe, dos clientes e do mercado, com o intuito de propor inovações, por meio de incentivo às novas idéias, cuidando para que as mesmas sejam colocadas em prática. Dessa maneira, a disposição para correr riscos e aprender com os próprios erros mostram-se comportamentos de especial relevância para a expressão da criatividade, uma vez que essa implica em lidar com o desconhecido, de forma a também implicar na geração de mudanças no local de trabalho e na busca de aperfeiçoamentos constantes (Alencar, 1998). Tais vantagens justificam a importância da condução de estudos sobre criatividade no ambiente organizacional, principalmente aqueles voltados à identificação de fatores individuais que podem influenciar o rendimento do trabalhador e, consequentemente, a produtividade da empresa.

Dentre esses fatores individuais, cuja influência amplia-se no sentido da realização pessoal, encontram-se os estilos de pensar e criar, resultantes da confluência de diversos fatores individuais e ambientais na expressão criativa do sujeito. De acordo com o modelo que busca explicar a autorrealização criativa, desenvolvido por Wechsler (1999), os estilos de criar estariam localizados na intersecção de dois grandes conjuntos: as habilidades cognitivas e as características da personalidade, ambos localizados dentro de um conjunto maior, o ambiente. Esses estilos seriam vistos, segundo Kirton (1994), como "preferências cognitivas consistentes e estáveis, que se manifestam em qualquer situação, envolvendo criatividade, solução de problemas e tomada de decisão" (p.37), de maneira que a realização criativa seria resultado da combinação de aspectos sociais, afetivos e cognitivos.

O conceito de estilo de criar vem aparecendo na literatura com mais frequência somente na última década, sendo reconhecida a sua importância para a compreensão do funcionamento do indivíduo criativo, uma vez que evidencia a existência de várias maneiras de se expressar a criatividade (Wechsler, 1999). Desta forma, a conceituação do termo refere-se a maneiras preferenciais de pensar e agir em determinadas situações, de forma a caracterizar certas tendências no comportamento e sentimento da pessoa criativa, cujo conhecimento atua de modo a facilitar a compreensão do modo de agir da 
pessoa criativa dentro de um determinado ambiente (Wechsler, 2008). Sua importância também ampara-se na possibilidade de, por esse meio, permitir um melhor aproveitamento dos potenciais e conhecimentos das necessidades individuais (Sternberg \& Lubart, 1997).

Os estilos constituem-se em uma das facetas da personalidade e apontam diferenças mais qualitativas do que quantitativas entre as pessoas (López \& Casullo, 2000), de maneira que, por esse motivo, se torna essencial o reconhecimento da existência de vários estilos, atentando-se para o fato de que não existem estilos "certos" e estilos "errados", e sim estilos individuais que diferem de uma pessoa para outra, conforme salientado por Sternberg (1997). Deve-se ressaltar que nenhum estilo é melhor, uma vez que eles apenas demonstram a forma como a pessoa utiliza a sua criatividade, permitindo compreender o funcionamento dos indivíduos, visto que os mesmos não representam um conjunto de capacidades e sim um conjunto de preferências (Nakano, 2010), não sendo usados para predizer o grau de criatividade (nível), mas sim sua natureza (Lubart, 2007). Dessa forma, ao elaborar um estudo dos estilos de pensar e criar dentre diferentes cargos organizacionais, encara-se a possibilidade de profissionais constituírem-se em agentes de mudanças e facilitadores de comportamentos organizacionais criativos, destacando-se, dentre as habilidades importantes, a existência de uma certa margem de liberdade para os comportamentos dos profissionais, incluindo-se a expressão criativa (Sousa \& Santos, 1999). Assim, "a compreensão dos estilos da pessoa criativa e seus modos preferenciais de pensar e criar em ambientes profissionais pode nos trazer importantes informações sobre aspectos ou formas de liderança organizacional" (Wechsler, 2006b, p. 217). Ainda de acordo com a autora, o conhecimento dos estilos poderia permitir uma melhor orientação profissional dos indivíduos segundo seus modos preferenciais de pensar e se comportar.

Vários foram os estudos brasileiros que buscaram identificar os estilos de pensar e criar em diferentes amostras e com diferentes objetivos. Dentre eles pode-se destacar o estudo de Wechsler (2006a), o qual investigou o estilo de 1752 adultos, com idades entre 17 e 70 anos, verificando a influencia das variáveis sexo e idade. Seus resultados indicaram que mulheres e homens tendem a possuir estilos diferentes, sendo os homens mais cautelosos e inconformistas que as mulheres. O estilo inconformista-transformador esteve mais frequentemente presente em indivíduos com idade superior a 24 anos. No 
contexto escolar, Siqueira e Wechsler (2004) investigaram a relação entre estilos de pensar e criar e desempenho escolar em uma amostra composta por 152 estudantes do Ensino Médio. Neste estudo, os estilos sensibilidade interna e externa e síntese humorística mostraram-se mais relacionados ao desempenho escolar, sendo os primeiros melhor pontuados pelas meninas, confirmando a hipótese das autoras de que as meninas se adaptam mais facilmente do que os meninos em sala de aula, dado o fato de apresentarem maior sensibilidade interna e externa como estilo criativo, o que possibilita a elas melhor rendimento acadêmico. Ainda nesse contexto, Homsi (2006) verificou que estudantes universitários $(n=126)$ das áreas de humanas, exatas e biológicas apresentavam estilos diferenciados, sendo o sexo feminino mais cauteloso e conservador do que o masculino, tendo, este último, apresentado humor em nível maior. A área biológica apresentou resultados significativamente maiores para o estilo pensamento-divergente e síntese humorística, sendo que a área de humanas destacou-se no estilo cauteloso-reflexivo.

Também verificando a influência do curso universitário, Nakano, Santos, Zavarize, Wechsler e Martins (2010) avaliaram o estilo de pensar e criar de 439 estudantes, dos cursos de Administração e Psicologia. Os resultados demonstraram que as variáveis gênero e curso não apresentaram influência significativa nos estilos, mas somente a interação entre elas no estilo relacionaldivergente. Em relação aos cursos, notou-se que estudantes do gênero feminino do curso de Administração apresentaram médias mais altas que as estudantes do curso de Psicologia, nos estilos inconformista-transformador, lógico-objetivo e relacional-divergente. Por sua vez, as estudantes de Psicologia apresentaram maiores médias nos estilos cauteloso-reflexivo e emocional-intuitivo, sendo que, em relação ao gênero masculino, estudantes do curso de Psicologia obtiveram médias mais altas que os do curso de Administração em todos os estilos avaliados, com exceção do emocional-intituitivo, no qual a pontuação foi bastante similar entre os estudantes dos dois cursos.

Godoy, Ottati e Noronha (2009) buscaram explorar a relação entre preferências profissionais e os estilos de pensar em criar, em 65 estudantes de graduação em Psicologia. Os resultados demonstraram que as maiores médias foram obtidas nos estilos relacional-divergente e inconformista-transformador, tendo, o estilo cauteloso-reflexivo, se mostrado mais presente nos estudantes do primeiro semestre, se comparados com os do terceiro semestre e o estilo 
relacional-divergente, mais comum entre os estudantes com mais de 26 anos. Estudantes de Psicologia também foram foco da pesquisa de Nakano (2010), desenvolvida junto a 186 estudantes de três regiões do Brasil (nordeste, sul e sudeste). Os resultados demonstraram que, de uma forma geral, estudantes das regiões sul e nordeste apresentaram um perfil similar quanto aos estilos predominantes, com os estudantes da região sudeste se diferenciando dos demais. A análise da variância indicou que a variável cidade de moradia exerce influência significativa no estilo dos estudantes, concluindo-se, segundo a autora, que as diferenças encontradas podem estar refletindo padrões culturais diversos presentes na população brasileira. Por fim, no contexto organizacional, Mundim e Wechsler (2007) buscaram identificar os estilos de pensar e criar em 72 líderes organizacionais e subordinados. Os resultados mostraram diferenças nos estilos inconformista-transformador, investimento-intuitivo e ousadia-intuitiva, mais pontuados pelos líderes, de forma que, segundo as autoras, tais resultados permitiram a identificação de gerentes e subordinados criativos através do seu estilo de pensar e criar. Ainda nesse estudo, as mulheres destacaram-se no estilo sensibilidade interna e externa, confirmando os resultados obtidos por Siqueira e Wechsler (2004).

Dado o fato que a avaliação dos estilos de pensar e criar dos indivíduos nos permite conhecer mais sobre o seu potencial de criar e inovar em diferentes campos de atuação, possibilitando oferecer-lhes maiores oportunidades para o desenvolvimento e expressão da sua criatividade (Wechsler, 2008), o presente estudo teve como objetivo identificar o estilo preferencial de pensar e criar dos profissionais de uma empresa do ramo de distribuição de produtos farmacêuticos, a fim de verificar a existência de diferentes estilos predominantes de pensar e criar, de acordo com as variáveis sexo, escolaridade e cargo ocupado.

\section{MÉTODO}

\section{Participantes}

A amostra foi composta por 40 profissionais que atuavam em uma empresa de distribuição de produtos farmacêuticos, localizada na cidade de João PessoaPB. Desses, 30 eram mulheres e 10 homens, com idades entre 17 anos e 35 anos $(M=24,5 ; D P=5,4)$ e escolaridade correspondente ao Ensino Médio $(n=14)$ e Superior $(n=26)$. Os participantes foram divididos em dois grupos, sendo o 
primeiro formado por profissionais que trabalhavam na área fiscal e contábil $(n=20)$, e outro grupo de profissionais que exerciam cargos na área de vendas $(n=20)$, detalhados na Tabela 1.

\section{Tabela 1.}

Descrição da amostra por grupo.

\begin{tabular}{|c|c|c|c|}
\hline Cargo & Idade & Sexo & Escolaridade \\
\hline Analista contábil jr. & 35 & $\mathrm{~F}$ & Superior completo \\
\hline Analista financeiro jr. & 35 & $\mathrm{~F}$ & 20 grau completo \\
\hline Analista financeiro jr. & 29 & $\mathrm{~F}$ & Superior completo \\
\hline Assistente contábil jr. & 28 & $\mathrm{~F}$ & 20 grau completo \\
\hline Analista financeiro jr. & 33 & $\mathrm{~F}$ & Superior completo \\
\hline Assistente contábil senior & 27 & $\mathrm{~F}$ & Superior completo \\
\hline Analista fiscal jr. & 18 & M & 20 grau completo \\
\hline Analista contábil sénior & 22 & M & Superior incompleto \\
\hline Auxiliar fiscal sénior & 34 & $\mathrm{~F}$ & Superior completo \\
\hline Assistente fiscal jr. & 19 & $\mathrm{~F}$ & Superior incompleto \\
\hline Analista financeiro jr. & 23 & $\mathrm{~F}$ & Superior incompleto \\
\hline Asisstente fiscal pleno & 34 & M & Superior completo \\
\hline Assistente contábil senior & 19 & M & Superior incompleto \\
\hline Analista contábil jr. & 19 & M & Superior incompleto \\
\hline Assistente contábil pleno & 20 & M & Superior incompleto \\
\hline Analista fiscal jr. & 35 & $\mathrm{~F}$ & Superior completo \\
\hline Assistente contábil senior & 20 & M & Superior completo \\
\hline Analista financeiro jr. & 25 & $\mathrm{~F}$ & Superior completo \\
\hline Analista contábil jr. & 19 & $\mathrm{~F}$ & Superior completo \\
\hline Assistente fiscal jr. & 17 & M & Superior incompleto \\
\hline \multicolumn{4}{|c|}{ Grupo 2 - Área de vendas } \\
\hline Auxiliar adm. vendas jr. & 24 & M & Superior completo \\
\hline Auxiliar pós-vendas pleno & 28 & $\mathrm{~F}$ & Superior incompleto \\
\hline Auxiliar adm. vendas interna & 25 & $\mathrm{~F}$ & 20 grau completo \\
\hline Auxiliar adm. vendas interna & 22 & $\mathrm{~F}$ & Superior incompleto \\
\hline Analista adm. vendas senior & 21 & M & Superior incompleto \\
\hline Auxiliar adm. vendas interna & 27 & $\mathrm{~F}$ & 20 grau completo \\
\hline Analista adm. vendas jr. & 20 & $\mathrm{~F}$ & 20 grau completo \\
\hline Coordenador Adm. vendas & 21 & $\mathrm{~F}$ & Superior completo \\
\hline Auxiliar adm. vendas jr. & 27 & $\mathrm{~F}$ & Superior completo \\
\hline Auxiliar adm. vendas jr. & 23 & M & 20 grau completo \\
\hline Analista adm. vendas jr. & 27 & $\mathrm{~F}$ & 20 grau completo \\
\hline Auxiliar adm. vendas interna & 20 & $\mathrm{~F}$ & 20 grau completo \\
\hline Auxiliar adm. vendas jr. & 31 & $\mathrm{~F}$ & 20 grau completo \\
\hline Auxiliar adm. vendas interna & 21 & $\mathrm{~F}$ & 20 grau completo \\
\hline Assistente adm. vendas pleno & 29 & $\mathrm{~F}$ & Superior completo \\
\hline Analista adm. vendas jr. & 19 & $\mathrm{~F}$ & 20 grau completo \\
\hline Auxiliar adm. vendas interna & 19 & $\mathrm{~F}$ & 20 grau completo \\
\hline Assistente adm. vendas & 21 & $\mathrm{~F}$ & 20 grau completo \\
\hline Auxiliar adm. vendas interna & 21 & $\mathrm{~F}$ & Superior completo \\
\hline Analista financeiro jr. & 26 & $\mathrm{~F}$ & Superior completo \\
\hline
\end{tabular}




\section{Instrumento}

O instrumento utilizado no estudo foi a Escala de "Estilos de Criar e Pensar" de Wechsler (2006a), composta por 100 frases, positivas e negativas, que devem ser respondidas dentro de uma escala Likert de 6 pontos indo de "discordo totalmente" a "concordo totalmente". Pode ser aplicada de forma coletiva ou individual, sendo estimado o tempo de 30 minutos para resposta.

O instrumento encontra-se validado no Brasil para uso a partir dos 15 anos e autorizado pelo Conselho Federal de Psicologia. A correção da escala dá origem a cinco pontuações totais, em cada um dos estilos (cauteloso-reflexivo, inconformista-transformador, lógico-objetivo, emocional-intuitivo e relacionaldivergente), de forma a permitir a identificação do estilo preferencial do respondente.

\section{Procedimentos}

Inicialmente foi obtido o consentimento da empresa para que a pesquisa fosse aplicada. Posteriormente, foi solicitada à direção uma lista com os nomes e cargos dos funcionários que atuavam na área selecionadas (fiscal-contábil e vendas), a fim de que os mesmos pudessem ser convidados a participar na pesquisa. Aqueles que concordaram, assinaram o termo de consentimento livre e esclarecido, respondendo ao instrumento de forma individual, recebendo, posteriormente, o resultado da avaliação.

Os instrumentos foram corrigidos pelas pesquisadoras e analisados em relação à média, desvio padrão, pontuação mínima e máxima, em cada um dos cinco estilos, considerando-se a amostra total e os subgrupos (separados por sexo, escolaridade e cargo). Posteriormente os resultados foram comparados buscando-se verificar a existência de diferenças significativas de acordo com o Teste $t$ de diferenças de médias.

\section{RESULTADOS E DISCUSSÃO}

A primeira análise realizada visou a estimativa da média e desvio padrão para cada um dos estilos avaliados pelo instrumento, considerando-se a divisão dos participantes por sexo ( $F=$ feminino; $M=$ masculino), nível de escolaridade (Médio e Superior) e área de atuação ( $F / C=$ fiscal-contábil; $V=$ vendas), disponíveis na Tabela 2. 
Tabela 2.

Estatística descritiva por estilo, sexo, escolaridade e área de atuação.

\begin{tabular}{cccccccc}
\hline Estilo & Estatística & \multicolumn{2}{c}{ Sexo } & \multicolumn{2}{c}{ Escolaridade } & \multicolumn{2}{c}{ Área } \\
\hline \multirow{4}{*}{ CR } & & $\mathrm{F}$ & $\mathrm{M}$ & Médio & Sup. & F/C & $\mathrm{V}$ \\
& $\mathrm{N}$ & 30 & 10 & 14 & 26 & 20 & 20 \\
& Média & 75,13 & 78,10 & 71,43 & 78,27 & 75,60 & 76,15 \\
IT & D.P. & 14,72 & 12,67 & 11,1 & 15,19 & 15,34 & 13,22 \\
& Média & 157,46 & 152,83 & 156,52 & 156,12 & 155,25 & 157,25 \\
LO & D.P. & 12,34 & 16,78 & 7,35 & 16,01 & 17,94 & 7,08 \\
& Média & 49,07 & 47,05 & 48,16 & 48,85 & 47,60 & 49,50 \\
EI & D.P. & 5,14 & 5,16 & 4,97 & 5,33 & 5,06 & 5,21 \\
& Média & 27,9 & 28,3 & 27,86 & 28,08 & 28,30 & 27,70 \\
RD & D.P. & 4,59 & 4,27 & 5,33 & 4,03 & 3,55 & 5,30 \\
& Média & 36,9 & 35,8 & 36,43 & 36,73 & 36,50 & 4,11 \\
& D.P. & 3,44 & 3,22 & 3,27 & 3,52 & 36,75 & 2,57 \\
\hline
\end{tabular}

Legenda: $\mathrm{CR}=$ Cauteloso Reflexivo, IT=Inconformista Transformador, LO=Lógico Objetivo, $\mathrm{EI}=$ Emocional Intuitivo, $\mathrm{RD}=$ Relacional Divergente, $\mathrm{F} / \mathrm{C}=$ Fiscal/Contábil, $\mathrm{V}=$ Vendas.

De acordo com os resultados, pode-se verificar que, em relação à variável sexo, as mulheres apresentaram médias maiores que os homens nos estilos Inconformista-transformador, Lógico-objetivo e Relacional-divergente, ao passo que o sexo masculino se destacou nos estilos Cauteloso-reflexivo e Emocionalintuitivo. Tais resultados concordam parcialmente com os resultados relatados por Wechsler (2006a), segundo o qual os homens mostraram-se mais cautelosos e inconformistas que as mulheres, sendo que, este último estilo, no presente estudo, foi melhor pontuado pelas mulheres. Nesse mesmo sentido, os resultados de Homsi (2006) indicaram que o sexo feminino tendeu a se mostrar mais cauteloso e conservador do que o masculino. Entretanto, os resultados apresentados na Tabela 2 contradizem os achados de Nakano et al (2010), cuja pesquisa com estudantes universitários apontou médias mais altas obtidas pelas mulheres em todos os estilos investigados, com exceção do relacionaldivergente, no qual os homens se destacaram, bem como os resultados de Siqueira (2001) e Mundim (2004), em cujas pesquisas as mulheres apresentaram escores significativamente mais altos no estilo sensibilidade interna e externa, mostrando-se mais atentas aos aspectos emocionais.

Considerando-se o nível de escolaridade dos participantes, verifica-se que o grupo composto por profissionais que possuem nível de escolaridade correspondente ao Ensino Superior apresentaram médias mais altas nos estilos Cauteloso-reflexivo, Lógico-objetivo, Emocional-intuitivo e Relacional-divergente, 
sendo que os participantes com Ensino Médio destacaram-se somente no estilo Inconformista-transformador (média ligeiramente superior que o outro grupo). Também estudantes universitários obtiveram médias mais altas no estilo cauteloso-reflexivo no estudo de Homsi (2006) e Nakano et al (2010), sendo que, neste último, destacaram-se ainda no estilo emocional-intuitivo, concordando com os resultados encontrados. Parcialmente concordam com os resultados apresentados por Godoy, Ottati e Noronha (2009), dado o fato de se destacar o estilo relacional-divergente, embora as autoras tenham relatado maiores médias no estilo inconformista-transformador, o qual foi mais bem pontuado, na presente pesquisa, pelos estudantes do Ensino Médio. Interessantemente, nenhum estudo encontrado na literatura brasileira investigou os estilos de pensar e criar tendo como foco diferenças devido a nível de escolaridade sendo, a maior parte deles, voltada ao estudo de um único nível educacional. Os resultados ainda demonstraram que os profissionais que atuam na área de vendas apresentam médias mais altas que os profissionais da área fiscal/contábil em todos os estilos avaliados pelo instrumento.

Diante das aparentes diferenças de médias, a Análise Multivariada da Variância foi empregada a fim de verificar a influência das variáveis sexo, nível de escolaridade e área de atuação, bem como suas interações, nos resultados dos participantes, cujos resultados encontram-se disponibilizados na Tabela 3. Salienta-se que a interação as três variáveis não foi possível de ser estimada dada a não existência de participantes em todas as condições.

Os resultados demonstraram que somente o estilo lógico-objetivo mostrouse influenciado pela área de atuação ( $F=4,745, p \square 0,037)$, sendo que todas as demais influências não mostraram significativas. Assim, pode-se afirmar que, na presente amostra, as variáveis sexo e nível de escolaridade não exerceram influência nos estilos de pensar e criar dos participantes, assim como todas as demais interações. Em relação ao nível de escolaridade nenhum estudo investigando a influência dessa variável nos estilos de pensar e criar foi encontrado na literatura. 
Tabela 3.

Análise da Variância para área de atuação, sexo, nível de escolaridade e estilo de pensar e criar.

\begin{tabular}{|c|c|c|c|c|}
\hline $\begin{array}{c}\text { Variável } \\
\text { independente }\end{array}$ & $\begin{array}{c}\text { Variável } \\
\text { dependente }\end{array}$ & $\begin{array}{c}\text { Médias ao } \\
\text { quadrado }\end{array}$ & $\mathrm{F}$ & $p$ \\
\hline \multirow[t]{5}{*}{ Área } & $\mathrm{CR}$ & 252,683 & 1,212 & 0,279 \\
\hline & IT & 119,991 & 0,590 & 0,448 \\
\hline & LO & 121,496 & 4,745 & $0,037^{*}$ \\
\hline & $\mathrm{EI}$ & 0,025 & 0,001 & 0,974 \\
\hline & RD & 10,413 & 0,845 & 0,365 \\
\hline \multirow[t]{5}{*}{ Sexo } & $\mathrm{CR}$ & 34,011 & 0,163 & 0,689 \\
\hline & IT & 7,870 & 0,039 & 0,845 \\
\hline & LO & 4,341 & 0,170 & 0,683 \\
\hline & $\mathrm{EI}$ & 0,422 & 0,018 & 0,894 \\
\hline & RD & 0,163 & 0,013 & 0,909 \\
\hline \multirow[t]{5}{*}{ Escolaridade } & CR & 234,341 & 1,124 & 0,297 \\
\hline & IT & 4,695 & 0,023 & 0,880 \\
\hline & LO & 10,083 & 0,394 & 0,535 \\
\hline & $\mathrm{EI}$ & 0,565 & 0,024 & 0,877 \\
\hline & RD & 2,022 & 0,164 & 0,688 \\
\hline \multirow[t]{5}{*}{ Área * Sexo } & $\mathrm{CR}$ & 295,781 & 1,419 & 0,242 \\
\hline & IT & 56,915 & 0,280 & 0,600 \\
\hline & LO & 79,394 & 3,101 & 0,088 \\
\hline & EI & 2,788 & 0,120 & 0,731 \\
\hline & RD & 9,778 & 0,793 & 0,380 \\
\hline \multirow[t]{5}{*}{ Área * Escolaridade } & CR & 0,192 & 0,001 & 0,976 \\
\hline & IT & 144,338 & 0,709 & 0,406 \\
\hline & LO & 51,426 & 2,009 & 0,166 \\
\hline & EI & 4,798 & 0,207 & 0,652 \\
\hline & RD & 24,442 & 1,983 & 0,168 \\
\hline \multirow[t]{5}{*}{ Sexo $*$ Escolaridade } & $\mathrm{CR}$ & 6,303 & 0,030 & 0,863 \\
\hline & IT & 164,985 & 0,811 & 0,374 \\
\hline & LO & 20,853 & 0,814 & 0,373 \\
\hline & $\mathrm{EI}$ & 0,105 & 0,005 & 0,947 \\
\hline & RD & 5,981 & 0,485 & 0,491 \\
\hline
\end{tabular}

A constatação da igualdade criativa entre homens e mulheres reforça uma das teorias defendidas na literatura científica acerca da hipótese de não existência de diferenças devido ao gênero na criatividade, fenômeno que vem sendo chamado de androgenia psicológica. Nesse sentido, alguns autores 
argumentam que os indivíduos criativos escapariam, em certa medida, ao rígido estereótipo dos papéis em função do gênero (Aranha, 1997; Candeias, 2008; De la Torre, 2005; Montuori \& Purser, 1995), de maneira que indivíduos criativos possuiriam uma capacidade de apresentar uma série de características pessoais favorecedoras da criatividade, independentemente do gênero a que pertencem, se assemelhando mais entre si do que em função do pertencimento a um ou outro grupo. Nesse sentido, dado o fato de que os estilos não se constituem em estilos certos ou errados, e sim em diferenças qualitativas entre as pessoas (López \& Casullo, 2000; Sternberg, 1997), os resultados encontrados apontam para o fato de que, na presente amostra, homens e mulheres não apresentam estilos predominantes, que poderiam tipificar um ou outro gênero. Tal dado será mais bem investigado posteriormente, através do levantamento da frequência com que cada estilo aparece (disponível na Tabela 3).

Entretanto, embora no estudo atual diferenças significativas de gênero não tenham sido observadas, convém salientar que outros estudos relataram diferenças devido a essa variável em relação aos estilos de pensar e criar (Cheng, Kim \& Hull, 2010; Homsi, 2006; Mundim \& Wechsler, 2007; Siqueira \& Wechsler, 2004), inclusive os estudos apresentados no próprio manual do instrumento. Na amostra de padronização, composta por 1752 participantes, com idades entre 17 e 72 anos, Wechsler (2006a) encontrou influência significativa para a variável sexo em quatro dos cinco estilos investigados, com exceção do estilo relacional divergente. Diferenças devido ao sexo também foram relatadas em outros estudos voltados à investigação de outros tipos de estilos, tais como os estilos cognitivos (Martins, Santos \& Bariani, 2005) ou estilos de liderança (Sonoo, Hoshino \& Vieira, 2008), de forma que, por esse motivo, cautela na interpretação dos resultados apresentados, bem como a recomendação de novos estudos são incentivados, a fim de que os mesmos possam, ou não, ser confirmados.

Uma segunda análise, com o objetivo de verificar a existência de um estilo predominante, de acordo com cada uma das áreas investigadas, gênero e nível de escolaridade, foi realizada a partir do levantamento do estilo de cada um dos participantes. O procedimento adotado seguiu as recomendações contidas no manual do instrumento, as quais apontam que, a partir da comparação entre as pontuações obtidas nos cinco estilos, torna-se possível conhecer o estilo predominante de cada participante, sendo aquele mais pontuado pelo mesmo. 
Tal classificação foi possível a partir da transformação dos escores brutos em escores padronizados, de acordo com as tabelas constantes no manual (divididas por sexo e idade). Dessa forma, encontra-se fornecida na Tabela 4 a frequência e porcentagem de cada estilo predominante nos grupos estudados. Convém salientar que a porcentagem em cada categoria ultrapassa o total de $100 \%$ devido ao fato de alguns indivíduos terem obtido pontuação igual em mais de um estilo, tendo-se optado por considerar os dois como predominantes. Nesse caso, para cálculo da porcentagem considerou-se o número total de sujeitos em cada categoria (feminino $=29$, masculino $=11$, ensino médio $=14$, ensino superior $=26$, grupo contábil/fiscal=20, grupo vendas=20) e não o número total de enquadres.

Tabela 4.

Frequência de estilo predominante por área de atuação, sexo e escolaridade.

\begin{tabular}{ccccccccccccc}
\hline Estilo & \multicolumn{4}{c}{ Sexo } & \multicolumn{4}{c}{ Escolaridade } & \multicolumn{4}{c}{ Área de atuação } \\
\hline & & F & \multicolumn{1}{c}{ M } & \multicolumn{1}{c}{ MÉDIO } & \multicolumn{3}{c}{ SUP. } & F / C & V \\
CR & 0 & 0 & 0 & 0 & 0 & 0 & 0 & 0 & 0 & 0 & 0 & 0 \\
IT & 8 & 27,5 & 1 & 32,0 & 3 & 21,4 & 6 & 23,0 & 5 & 25,0 & 4 & 20,0 \\
LO & 18 & 62,0 & 8 & 72,7 & 8 & 57,1 & 18 & 69,2 & 12 & 60,0 & 14 & 70,0 \\
EI & 2 & 6,8 & 1 & 18,2 & 2 & 14,3 & 1 & 3,8 & 1 & 5,0 & 2 & 10,0 \\
RD & 4 & 13,8 & 1 & 18,2 & 3 & 21,4 & 2 & 7,6 & 2 & 10,0 & 3 & 15,0 \\
Total & 29 & & 11 & & 14 & & 26 & & 20 & & 20 \\
\hline
\end{tabular}

Uma análise da Tabela 4 mostra que a maior parte da amostra estudada apresenta como estilo predominante o Lógico-objetivo, independente do sexo (62,0\% das mulheres foram classificadas nesse estilo e $72,7 \%$ dos homens), do nível de escolaridade (com 57,1\% dos profissionais com Ensino Médio e 69,2\% com Ensino Superior) e em relação à área de atuação (60,0\% dos participantes da área contábil/fiscal apresentando esse estilo predominante e 70,0\% dos profissionais da área de vendas). Assim, o que se pode afirmar é que, independente do gênero, área de atuação e nível de escolaridade, os participantes nessa amostra apresentam estilos semelhantes, quando divididos de acordo com essas variáveis, confirmando os resultados apontados pela Análise Multivariada da Variância, de acordo com a qual, essas variáveis não exerceram influência em nenhum dos estilos, somente no estilo lógico-objetivo de acordo com a área de atuação.

A descrição desse estilo, segundo o manual (Wechsler, 2006a), mostra que as pessoas que apresentam o estilo Lógico-objetivo podem ser descritas como 
pessoas que se caracterizam pelo pensamento lógico, racional e pragmático, que preferem trabalhar com tarefas já estruturadas nas quais existam soluções conhecidas. Gosta de seguir regras, sendo bastante persistente em suas ações, reflete bastante antes de agir, controla suas emoções e sentimentos, gosta de situações práticas e evita qualquer grau de improvisação. Apresenta dificuldade em trabalhos com grupos pois apresentam dificuldade em lidar com pessoas pouco objetivas. Podem alcançar inovações se unindo à pessoas com os estilos Inconformista-transformador ou Intuitivo-emocional para que se arrisque mais e dê maior espaço para as suas emoções.

Diante dessa descrição, pode-se verificar a adequação do estilo às atividades que são desenvolvidas cotidianamente nas duas áreas, que envolvem raciocínio lógico, matemático e lógico, além da necessidade de objetividade no trabalho, tanto em relação ao controle financeiro da instituição, como na necessidade de fechamento de vendas para a manutenção da empresa. Outra ressalva deve ser feita no sentido de destacar a não homogeneidade de nível de escolaridade de acordo com as áreas, dado o fato de que os cargos analisados não apresentavam perfil de escolaridade diferente, podendo-se notar participantes com os dois níveis educacionais atuando nas duas áreas. Nesse sentido, Wechsler (2009) destaca que vários estilos podem ser encontrados em pessoas criativas ou naquelas que se encontram liderando diferentes áreas, embora, na visão do senso comum, as pessoas criativas sejam descritas como aquelas que apresentam características mais relacionadas ao estilo inconformista-transformador. A autora ainda salienta que podemos encontrar indivíduos que produzem de outras formas ou estilos, de forma que tal percepção pode ser usada no sentido de quebrar o preconceito existente acerca de uma única forma de pensar que conduz à criatividade.

\section{CONSIDERAÇÕES FINAIS}

O que se pode afirmar é que existem, sem dúvidas, algumas características que podem identificar ou serem descritoras das pessoas criativas, demonstrando a importância de se estimular pensamentos e sentimentos, de forma a desenvolver talentos individuais e tornar o ambiente organizacional encorajador a novas idéias e local de desenvolvimento pessoal (Wechsler, 1999). Isso porque, de acordo com Wechsler (2001), pesquisas comprovaram a relevância 
do reconhecimento dos estilos de pensamento como forma essencial no estímulo do potencial individual.

Assim, Lubart (2007) destaca o fato de que os quadros profissionais têm impacto sobre a expressão criativa, pois podem tanto oferecer um ambiente favorável às condutas criativas como representar um freio considerável à criatividade, de modo que, ainda de acordo com o autor, no adulto, o tipo de atividade profissional determina, em parte, as possibilidades de exercer a criatividade. Nesse sentido, Candeias (2008) também afirma que os contextos podem inibir ou estimular a criatividade, pois mesmo que os recursos internos estejam presentes, se a pessoa não encontrar espaço ou ambiente em que possa propor idéias, a criatividade pode não se manifestar. Muitas vezes, os profissionais já ingressam em uma organização com uma idéia do perfil do profissional esperado pela empresa, ao qual, provavelmente, tendem a se moldar. Ao assim procederem, desrespeitam o estilo próprio, de forma que a expressão criativa fica dificultada, causando prejuízos tanto na esfera pessoal como profissional.

A importância de estudos sobre os estilos baseia-se, segundo Martins, Santos e Bariani (2005), no fato de que, em função dos estilos, os profissionais podem revelar melhor desempenho em diferentes situações. Um maior conhecimento sobre os estilos cognitivos poderia favorecer processos de rendimento profissional, respeitando as características individuais. Ainda de acordo com as autoras, seria altamente desejável um maior investimento em pesquisas que focalizem variáveis psicológicas, como os estilos cognitivos, uma vez que podem interferir na expressão criativa. De acordo com Wechsler (2009), "a compreensão dos estilos de pensar e criar pode fornecer importantes informações sobre as pessoas criativas, ao mesmo tempo em que permitiria a compreensão do papel que diferentes estilos podem desempenhar ao seu buscar o funcionamento eficaz de uma equipe, ou ainda, nas diferentes fases de elaboração e finalização de um produto criativo" (p.56). Nesse sentido, a autora ainda destaca a importância da combinação ou complementação de estilos para que uma organização possa se mostrar eficaz, dado o fato de que diferentes estilos podem se tornar fonte enriquecida da diversidade de opiniões, em oposição à ideia de que devem constituir-se em foco de discórdia em uma equipe. Tal combinação pode ser usada no sentido de favorecer o alcance de inovações em uma determinada organização. 
A carência de estudos que investigam essa temática no ambiente organizacional, constatada pela própria autora do instrumento utilizado, aponta para a necessidade de que "estudos futuros deverão ainda ser feitos para examinar melhor a influência do sexo e área profissional em determinados tipos de estilos de pensar e criar" (Wechsler, 2008, p.215), de forma que estudos que contemplem amostras maiores e mais diversificadas, bem como a investigação dos estilos de pensar e criar em empresas de outros ramos e outros locais, são incentivados, a fim de que melhor se possa compreender a influência dos estilos na criatividade profissional e organizacional. A ampliação das amostras poderá sanar uma das limitações do presente estudo, dada a restrição no número de participantes e sua composição, em sua maior parte, por indivíduos do sexo feminino, características que podem ter influenciado nos resultados obtidos.

\section{REFERÊNCIAS}

Alencar, E. M. L. S. (1998). Promovendo um ambiente favorável à criatividade nas organizações. Revista de Administração de Empresas, 38(2), 8-25.

Alencar, E. M. L. S. (2001). Criatividade e educação de superdotados. Petrópolis, RJ: Vozes.

Alencar, E. M. L. S. (2005). A gerência da criatividade. São Paulo: Makron Books. Alencar, E. M. L. \& Fleith, D. S. (2003). Barreiras à criatividade pessoal entre professores de distintos níveis de ensino. Psicologia Reflexão e Crítica, 16(1), 63-69.

Aranha, M. A. R. C. (1997). Creativity in students and its relation to intelligence an peer percetion. Interamerican Journal of Psychology, 31(2), 309-313.

Bruno-Faria, M. F., Veiga, H. M. S. \& Macêdo, L. F. (2008). Criatividade nas organizações: análise da produção científica nacional em periódicos e livros de Administração e Psicologia. Psicologia das Organizações e Trabalho, 8(1), 142-163.

Candeias, A. A. (2008). Criatividade: perspectiva integrativa sobre o conceito e sua avaliação. In M. F. Morais \& S. Bahia. Criatividade: Conceito, necessidades e intervenção (pp.41-64). Braga: Psiquilíbrios. 
Candeias, A. A., Rebelo, N., Silva, J., \& Mendes, P. (2011) Excelência vs. Competência: um desafio para a educação e o desenvolvimento profissional. In S. M. Wechsler \& T. C. Nakano (Orgs.). Criatividade no ensino superior: Uma perspectiva internacional (pp. 54-79). São Paulo: Vetor.

Cheng, Y., Kim, K. H., \& Hull, M. F. (2010). Comparisons of creative styles and personality types between American and Taiwanese college students and the relationship between creative potential and personality types. Psychology of Aesthetics, Creativity, and the Arts, 4(2), 103-112.

Correia, G. S., \& Dellagnelo, E. H. L. (2004). Avaliação do potencial da estrutura para o desenvolvimento da criatividade em uma indústria cerâmica catarinense. Em Anais do Encontro da Associação Nacional dos Programas de Pós-graduação e Pesquisa em Administração (pp. 28). Curitiba: ANPAD. Disponível em: http://anais.sepex.ufsc.br/anais_4/trabalhos/243.html. Acesso em 10/11/2011.

Crespo, M. L. F. (2004). Construção de uma medida de clima criativo em organizações. Estudos de Psicologia (Campinas), 21(2), 91-99.

De la Torre, S. (2005). Dialogando com a criatividade. São Paulo: Madras.

El-Murad, J., \& West, D.C. (2004). The definition and measurement of creativity: what do we know? Journal of Advertising Research, 44(2), 188-201.

Godoy, S., Ottati, F., \& Noronha, A.P.P. (2009). Interesse profissional e estilos de pensar e criar em estudantes de Psicologia. Boletim de Psicologia, 59(131), 191-207.

Gonçalvez, F. C., Fleith, D. S. \& Libório, A. C. O. (2011). Criatividade em aula: Percepção de alunos de dois estados brasileiros. Arquivos Brasileiros de Psicologia, 63(1), 22-30.

Homsi, S. H. V. (2006). Temperamento e sua relação com estilos de pensar e criar. Dissertação de Mestrado. Pontifícia Universidade Católica de Campinas.

Kirton, M. J. (1994). Adaptors and innovators: Styles of creativity and problem solving. London: Routledge.

Lins, M. J. S. C. \& Miyata, E. S. (2008). Avaliando a aprendizagem de criatividade em uma oficina pedagógica. Ensaio: Avaliação de Políticas Públicas em Educação, 16(60), 455- 468. 
López, M. P. S. \& Casullo, M. M. (2000). Ensino e estilo individual de aprendizagem. In M. P. S. López \& M. M. Casullo (Orgs). Estilos de personalidad: Una perspectiva iberoamericana (pp.11-15). Buenos Aires: Mino y Davila.

Lubart, T. (2007). Psicologia da criatividade. Porto Alegre: ArtMed.

Martinez, A. M. (2000). A criatividade nas organizações: O papel do líder. Universitas Psicologia, 1(1), 59-78.

Martinez, A. M. (2002). A criatividade na escola: Três direções de trabalho. Linhas Críticas, 8(15), 189-206.

Martins, R. M. M., Santos, A. A. A. \& Bariani, I. C. D. (2005). Estilos cognitivos e compreensão leitora em universitários. Paidéia, 15(30), 57-68.

Montuori, A., \& Purser, R.E. (1995). Deconstructing the lone genius myth: Toward a contextual view of creativity. Journal of Humanistic Pyschology, 35(3), 69-112.

Moraes, M.M. \& Lima, S.M.V. (2009). Estratégias para criar no trabalho: Proposição teórica e validação psicométrica de medida. Paidéia, 19(44), 367-377.

Mundim, M. C. B. (2004). Estilos de criar em lideres organizacionais. Dissertação de Mestrado. Pontifícia Universidade Católica de Campinas.

Mundim, M. C. B. \& Wechsler, S. M. (2007). Estilos de Pensar e Criar em gerentes organizacionais e subordinados. Boletim de Psicologia, 57(126), 15-32.

Nakano, T. C. (2005). Pesquisa em criatividade: Análise da produção científica do banco de teses da Capes (1996-2001). In G. P. Witter. Metaciência e Psicologia (pp.35-48). Campinas: Editora Alínea.

Nakano, T. C. (2010). Estilos de pensar e criar em estudantes de psicologia: diferenças regionais? Estudos e Pesquisas em Psicologia, 10(3), 682-699.

Nakano, T. C., Santos, E., Zavarize, S. F., Wechsler, S. M. \& Martins, E. (2010). Estilos de pensar e criar em universitários das áreas de humanas e sociais aplicadas: Diferenças por género e curso. Psicologia Teoria e Prática, 12(3), 120-134.

Nakano, T. C. \& Wechsler, S. M. (2007). Criatividade: Características da produção científica brasileira. Avaliação Psicológica, 6(2), 261-270.

Nicolas, A. M. N. (1999). Criatividade onde está? Catharsis, 4(23), 11-12. 
Oliveira, Z. M. F. (2007). Criatividade na formação do professor do curso de letras. Dissertação de Mestrado não publicada, Curso de Pós-Graduação em Educação, Universidade Católica de Brasília. Brasília, DF.

Oliveira, Z. M. F. (2010). Fatores influentes no desenvolvimento do potencial criativo. Estudos de Psicologia (Campinas), 27(1), 83-92.

Sakamoto, C. K. (2000). Criatividade: uma visão integradora. Psicologia: Teoria e Prática, 2(1), 50-58.

Siqueira, L. G. G. (2001). Estilos de criar e desempenho escolar. Dissertação de Mestrado. Pontifícia Universidade Católica de Campinas.

Siqueira, L. G. G. \& Wechsler, S. M. (2004). Estilos de pensar e criar de estudantes brasileiros e sua influência sobre o desempenho escolar. Revista Iberoamericana de Diagnóstico y Evaluación Psicológica, 18(2), 15-22.

Sonoo, C. N., Hoshino, E. F. \& Vieira, L. F. (2008). Liderança esportiva: Estudo da percepção de atletas e técnicos no contexto competitivo. Psicologia: Teoria e Prática, 10(2), 68-82.

Sousa, L. C. \& Santos, L. (1999). A relação entre estilos pedagógicos e desempenho escolar em Portugal. Psicologia Reflexão e Critica, 12(2), 331342.

Sternberg, R. (1997). Thinking styles. New York: Cambridge University Press.

Sternberg, R. J. \& Lubart, T. I. (1997). La creatividad en una cultura conformista: un desafío a las massas. Barcelona: Paidós.

Wechsler, S. M. (1999). Avaliação da criatividade: um enfoque multidimensional. In S. M. Wechsler \& R. S. L Guzzo. Avaliação psicológica: Perspectiva internacional (p.231-260). São Paulo: Casa do Psicólogo.

Wechsler, S. M. (2001). Criatividade na cultura brasileira: uma década de estudos. Psicologia: Teoria, Investigação e Prática, 6(1), 215-226, 2001.

Wechsler, S. M. (2006a). Estilos de Pensar e Criar (manual). Campinas: Impressão Digital do Brasil/LAMP.

Wechsler, S. M. (2006b). Estilos de pensar e criar: Impacto nas áreas educacional e profissional. Psicodebate: Psicologia, Cultura y Sociedad, 7, 207-218.

Wechsler, S. M. (2008). Criatividade: Descobrindo e encorajando. São Paulo: Editora Psy. 
Wechsler, S. M. (2009). Estilos de pensar e criar: Implicações para a liderança. In Z. G. Giglio, S. M. Wechsler \& D. Bragotto. Da criatividade a inovação (pp.39-60). Campinas, SP: Papirus.

Wechsler, S. M. \& Nakano, T. C. (2002). Caminhos para a avaliação da criatividade: Perspectiva brasileira. Em R. Primi. (2002). Temas em avaliação psicológica. (pp.103-115). São Paulo: Instituto Brasileiro de Avaliação Psicológica.

Wechsler, S. M. \& Nakano, T. C. (2003). Produção brasileira em criatividade: 0 estado da arte. Escritos sobre Educação, 2(2), 43-50.

Zanella, A. V. \& Titon, A. P. (2005). Análise da produção científica sobre criatividade em programas brasileiros de pós-graduação em psicologia (1994 -2001). Psicologia em Estudo, 10(2), 305-316.

Contato: tatiananakano@hotmail.com

Recebido em: 03/11/2011

Revisado em: 15/12/2011

Aceito em: 20/12/2011 